\title{
Structure and magnetism of Tm atoms and monolayers on W(110)
}

\author{
Corneliu Nistor, ${ }^{1,2}$ Aitor Mugarza, ${ }^{2}$ Sebastian Stepanow, ${ }^{1}$ and Pietro Gambardella ${ }^{1,2}$ \\ ${ }^{1}$ Department of Materials, ETH Zurich, $\mathrm{CH}-8093$ Zurich, Switzerland \\ ${ }^{2}$ Catalan Institute of Nanoscience and Nanotechnology (ICN2), UAB campus, E-08193 Barcelona, Spain
}

\author{
Kurt Kummer ${ }^{3}$ \\ ${ }^{3}$ European Synchrotron Radiation Facility (ESRF), F-38043 Grenoble, France
}

\author{
José Luis Diez-Ferrer, ${ }^{4}$ David Coffey, ${ }^{5,6}$ César de la Fuente,,${ }^{5,6}$ Miguel Ciria,,${ }^{5,6}$ and José I. Arnaudas ${ }^{4,6}$ \\ ${ }^{4}$ Laboratorio de Microscopias Avanzadas, Instituto de Nanociencia de Aragón, Universidad de Zaragoza, E-50018 Zaragoza, Spain \\ ${ }^{5}$ Instituto de Ciencia de Materiales de Aragón, Consejo Superior de Investigaciones Científicas, E-50009 Zaragoza, Spain \\ ${ }^{6}$ Departamento de Física de la Materia Condensada, Universidad de Zaragoza, E-50071 Zaragoza, Spain \\ (Received 10 February 2014; revised manuscript received 30 July 2014; published 25 August 2014)
}

\begin{abstract}
We investigated the growth and magnetic properties of Tm atoms and monolayers deposited on a W(110) surface using scanning tunneling microscopy and x-ray magnetic circular and linear dichroism. The equilibrium structure of Tm monolayer films is found to be a strongly distorted hexagonal lattice with a Moiré pattern due to the overlap with the rectangular W(110) substrate. Monolayer as well as isolated Tm adatoms on W present a trivalent ground-state electronic configuration, contrary to divalent gas phase Tm and weakly coordinated atoms in quench-condensed Tm films. Ligand field multiplet simulations of the x-ray absorption spectra further show that $\mathrm{Tm}$ has a $\left|J=6, J_{z}= \pm 5\right\rangle$ electronic ground state separated by a few meV from the next lowest substates $\left|J=6, J_{z}= \pm 4\right\rangle$ and $\left|J=6, J_{z}= \pm 6\right\rangle$. Accordingly, both the Tm atoms and monolayer films exhibit large spin and orbital moments with out-of-plane uniaxial magnetic anisotropy. X-ray magnetic dichroism measurements as a function of temperature show that the Tm monolayers develop antiferromagnetic correlations at about $50 \mathrm{~K}$. The triangular structure of the Tm lattice suggests the presence of significant magnetic frustration in this system, which may lead to either a noncollinear staggered spin structure or intrinsic disorder.
\end{abstract}

DOI: 10.1103/PhysRevB.90.064423

PACS number(s): 75.70.Ak, 75.50.Ee, 68.55.-a, 32.30.Rj

\section{INTRODUCTION}

Interest in low-dimensional rare-earth (RE) magnetic structures has grown steadily in recent years, focusing on single atoms as model quantum spin systems [1-5] as well as ultrathin films that display either ferromagnetic [6-9] or antiferromagnetic [10] order when grown on nonmagnetic substrates. Ultrathin RE magnetic films and multilayers have also attracted attention due to their ability to induce perpendicular magnetic anisotropy in adjacent transition-metal layers [11], control magnetic damping in spin valve devices [12,13], as well as fabricate materials with higher magnetization compared to transition-metal alloys $[14,15]$.

The interest in studying the magnetism of RE adatoms and monolayers (ML) stems from the localized character of the $4 f$ states, which protects them from hybridization effects and preserves atomiclike spin and orbital magnetic moments. The extended $5 d 6 s$ valence electron states of the RE atoms, on the other hand, couple with the partially filled $4 f$ orbitals and hybridize with the surface valence electrons of the substrate. This affects the exchange interaction and, hence, the type of magnetic order and transition temperatures in ML structures [16-18]. Additionally, combined with hybridization effects, symmetry breaking and epitaxial strain influence the magnetic anisotropy and magnetoelastic interactions [19], giving rise to a different magnetic behavior compared to that of thick films.

In contrast to transition-metal adatoms, which have been extensively investigated using the anomalous Hall effect [20], $\mathrm{x}$-ray magnetic circular dichroism (XMCD) [21-25], photoemission [26], and scanning tunneling microscopy (STM)
[25,27-29], there are very few experimental studies on the magnetism of RE atoms on surfaces. To our knowledge, only $\mathrm{Ce}$ [1,2], Gd [3], and Ho [5] impurities have been investigated, mainly by STM. Theoretical studies are also limited to $\mathrm{Gd}$ adatoms and dimers on $\mathrm{CuN} / \mathrm{Cu}$ substrates [4] and Ho adatoms on $\operatorname{Pt}(111)$ [5].

RE thin films deposited on crystalline magnetic and nonmagnetic transition-metal substrates have been previously studied by spin-polarized photoelectron [9,30,31] and Auger [32] spectroscopies, electron capture spectroscopy [6], as well as by linear and circular x-ray dichroism [33-35] and spin-polarized STM [8,36-38]. Gd is the RE element that has been more extensively studied in the form of ultrathin films, mostly because it exhibits a single ferromagnetic phase in the bulk with the order transition close to room temperature $[7,39,40]$. Extrapolating the thickness dependence of the Curie temperature leads to vanishing magnetic order for Gd films thinner than 3 monolayers (ML) [7]. Similarly, in $\mathrm{Ho} / \mathrm{W}(110)$ thin films, resonant magnetic soft $\mathrm{x}$-ray and neutron diffraction experiments [10] have shown that the helical antiferromagnetic ordering temperature $T_{N}$ decreases with the film thickness following a modified power law with an offset of about $11 \mathrm{ML}$, below which the long-period basal plane antiferromagnetic structure is not possible. It is worth mentioning that the decrease of the order temperature with thickness in these RE is stronger than in the case of transition-metal ferromagnets [41,42]. Moreover, below the critical temperature, bulk RE metals display magnetically ordered phases of different complexity, depending on the element and on the temperature range [43]. These examples 
show that the magnetic structure of RE ultrathin films is a nontrivial issue, which, furthermore, has not been investigated in a systematic way across the lanthanide series.

Tm represents an interesting case since it is the only heavy RE which orders magnetically along the $c$ axis of the bulk hcp crystal structure. Between the Néel temperature $T_{N}=58$ and $40 \mathrm{~K}$, the magnetic moments are ferromagnetically ordered within the hcp basal-plane layers and have an incommensurate sinusoidal modulation along the $c$ axis. The easy axis is parallel to the $c$ axis, owing to strong crystal-field anisotropy. Upon decreasing the temperature, the propagation wave number along the $c$ axis increases and, below $\sim 30 \mathrm{~K}$, a ferrimagnetic structure with a seven-layer repeat distance develops: the magnetic moments point up along the $c$ axis in three layers and down in the consecutive four layers. Although the reduction of thickness along the $c$ axis could drastically influence the magnetic behavior of this element, no structural and magnetic characterization of thin or ultrathin layers of Tm has been reported to date. In this work, we investigate the growth and magnetic properties of Tm adatoms and ML films deposited on a single-crystal W(110) surface.

\section{EXPERIMENTS}

The growth of Tm on W(110) was investigated by means of STM and low-energy electron diffraction (LEED) at the University of Zaragoza. Sample preparation was carried out in an ultrahigh vacuum chamber with base pressure of $\sim 10^{-10}$ Torr, which included an $e$-beam heating station, LEED, Auger electron spectroscopy, and an $e$-gun used to evaporate Tm (purity 99.99\%) by heating a tungsten crucible holding Tm pieces. Prior to the evaporation of Tm, the W(110) single-crystal surface was cleaned by cycles of annealing in oxygen atmosphere and flashes at temperatures around $2400 \mathrm{~K}$. The oxygen partial pressure was reduced from $\sim 10^{-6}$ Torr to $\sim 10^{-9}$ Torr between subsequent annealing cycles of $30 \mathrm{~min}$ at $1500 \mathrm{~K}$. During Tm evaporation, the pressure remained below $\sim 10^{-9}$ Torr, allowing deposition rates as low as $1 \mathrm{ML} / \mathrm{min}$. The vacuum in the STM chamber was better than $\sim 10^{-11}$ Torr. The LEED image [see Fig. 1(a)] shows the reciprocal lattice of the W(110) surface with sharp spots without trace of carbon surface reconstructions. The state of the bare $\mathrm{W}$ surface was checked by STM [Fig. 1(b)], which showed atomically flat terraces separated by monoatomic steps $(\sim 2.21 \AA)$. The inset of Fig. 1(b) shows an enlarged area with a density of impurities as low as $0.3 \%$, comparable with other works [44].
The Tm depositions were carried out at room temperature and the samples annealed at temperatures between 500 and $1100 \mathrm{~K}$ for $10 \mathrm{~min}$. The annealing conditions and the coverage are key factors that determine the structure of the samples. For short deposition times at low flux and annealing temperatures below $\sim 1000 \mathrm{~K}$, we obtain coverages of $\sim 2 \%$, consisting mainly of isolated Tm adatoms, as shown in Fig. 1(c). Some larger objects, which we identify as dimers and trimers since they occasionally split during the interaction with the STM tip, are also found, but represent a small percentage of the total coverage. LEED images of these lowcoverage samples do not show diffraction spots additional to those of the W(110) surface. It is remarkable that low-coverage Tm deposits annealed at temperatures as high as $1000 \mathrm{~K}$ do not coalesce but remain as isolated adatoms, possibly due to the influence of repulsive Ruderman-Kittel-Kasuya-Yosida (RKKY) [45] or dipolar interactions, as we will report in a separate publication [46]. Larger flux rate and annealing temperatures of $\sim 1100 \mathrm{~K}$ give rise to heteroepitaxial growth with the formation of one-monolayer-thick islands of Tm on W(110), as determined by direct STM observation as well as by LEED analysis.

The electronic and magnetic characterization was carried out by means of x-ray magnetic circular and linear dichroism, XMCD and XMLD, respectively. The measurements were performed at the ID08 beamline of the European Synchrotron Radiation Facility, where the samples were grown in situ according to the procedure described above and probed by STM and LEED. Following preparation, the samples were transferred in ultrahigh vacuum into the $\mathrm{x}$-ray absorption measurement chamber, which provides a focused $x$-ray beam with tunable energy, $99 \pm 1 \%$ circular and linear polarization, and a $0.1 \times 1 \mathrm{~mm}^{2} \mathrm{x}$-ray spot at full width at half maximum. The samples were mounted vertically on a variable-temperature (6-300 K) rotary stage holder which permitted rotation of the substrate around the vertical axis. A superconducting magnet allowed the application of magnetic fields of up to $5 \mathrm{~T}$ along the x-ray beam direction. X-ray absorption spectra (XAS) were measured at the $M_{4,5}$ absorption edges of Tm (corresponding to $3 d$ to $4 f$ core-to-valence electronic transitions) in the total electron yield mode by recording the drain current of the sample as a function of photon energy. The drain current was normalized by the photocurrent of a gold grid reference placed between the last refocusing mirror and the sample. The linearly polarized XAS were measured by aligning the
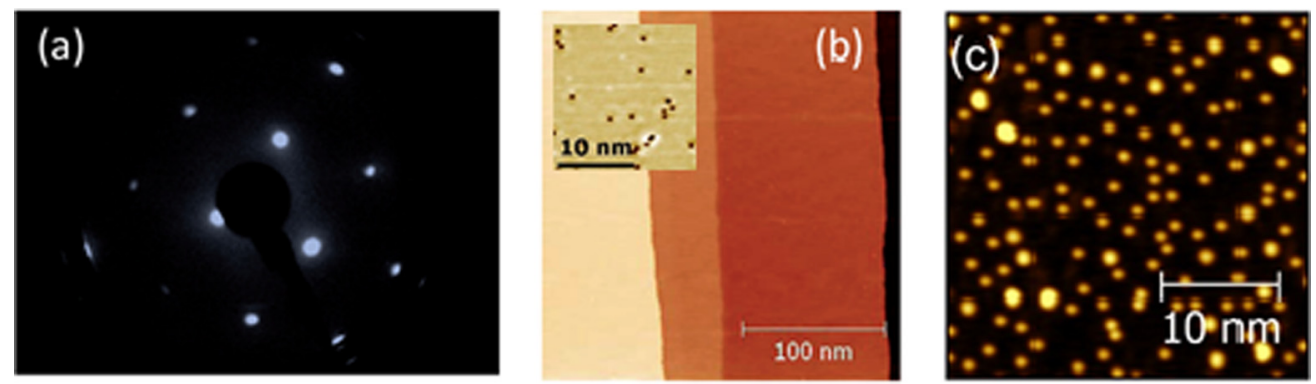

FIG. 1. (Color online) (a) LEED image taken on the W(110) surface after cleaning using the procedure described in the text, (b) STM images of W(110) showing terraces, (inset) detail of residual carbon impurities remaining after the cleaning procedure, (c) STM image of Tm adatoms (coverage $\sim 2 \%$ ) on the $\mathrm{W}(110)$ surface. The larger objects correspond to Tm dimers and trimers. 
electric field vector of the $\mathrm{x}$ rays to the vertical $\left(I^{v}\right)$ and horizontal $\left(I^{h}\right)$ axis, with the beam incident at an angle $\theta=70^{\circ}$ with respect to the sample normal. The XMCD spectra were obtained by taking the XAS difference for parallel $\left(I^{+}\right)$and antiparallel $\left(I^{-}\right)$alignment of the photon helicity with the applied magnetic field. During the measurements, the pressure in the cryostat was about $2 \times 10^{-10}$ mbar and likely lower in the sample region surrounded by the cryogenic shields. No changes of the XAS line shape, due to either the X-ray beam or contamination, were observed within the few hours required to measure one sample. The XAS data were simulated using ligand field atomic multiplet calculations $[47,48]$ as described in Refs. [49,50].

\section{STRUCTURAL CHARACTERIZATION}

The (110) surface of $\mathrm{W}$ offers the opportunity of studying the magnetic behavior of ordered RE overlayers on top of a conductive but nonmagnetic substrate. In general, the body-centered-cubic crystals of refractory metals such as W and Mo promote the two-dimensional layer growth of RE with no intermixing [51-53], with the (110) surface yielding morphologies with low corrugation.

It is known that below the monolayer coverage rare-earth metals adsorbed on W(110) or Mo(110) show superstructures consisting of wires with the axis along the W[110] direction and hexagonal structures [51,52,54,55]. Figure 2(a) shows a LEED pattern, taken at $145 \mathrm{eV}$, of a deposit that resulted in an ordered superstructure. This LEED image shows the spots of the W(110) surface and six spots characteristic of the (0001) surface of the RE hcp structure, but also satellite spots indicating the existence of a superstructure. The image is quite similar to that reported by Kolaczkiewicz et al. [51] indexed as a mix of $c(5 \times 3)$ and $(5 \times 2)$ structures and observed for $\mathrm{Gd}$ and Eu grown on W(110). Although in some cases we obtained such reconstructions, we were able, by finely adjusting the flux rate and the annealing temperature, to obtain samples that do not show them. The XMCD measurements reported in Secs. IV and $\mathrm{V}$ were performed on unreconstructed Tm monolayers. Figure 2(b) shows a LEED image in which the six sharp spots characteristic of the (0001) surface of the RE hcp structure and the spots of the W(110) surface are observed simultaneously, with no trace of satellites. The $b$ direction [10 $\overline{1} 0$ ] coincides with the [1ㅣㅣ direction of the (110) substrate, similar to the
Nishiyama-Wasserman orientation already observed in other RE/W(110) systems [56,57]. In Fig. 2(c), we display a STM image of one of these nonreconstructed samples, taken in an area with a monolayer of Tm showing a detail of the Tm adatoms with atomic resolution. The lattice parameter measured along [001] is $4.08 \AA$, while for the two other sides of the isosceles triangle we obtain $3.92 \AA$. This means that the Tm ML displays a fairly distorted hexagonal structure, forming in fact a rhombic or isosceles triangular lattice. Thus, the lattice mismatch between Tm and $\mathrm{W}$ is large enough to produce in the first monolayer of thulium an asymmetric distortion of the hcp structure that, with respect to the bulk lattice parameters, is compressed along the [1 10$] \mathrm{W}$ by $1 \%$ and expanded along [001] $\mathrm{W}$ by about $15 \%$.

The image also reveals the formation of a Moiré pattern caused by the overlap of this triangular Tm lattice with the rectangular (110) W surface, which should have a lattice parameter of $3.173 \AA$, a value slightly larger than the bulk one (3.165 $\AA$ ). We obtain a Tm:W coincidence match of 2:3 and 7:9 along the [110] and [001] $\mathrm{W}$ directions, respectively. Taking into account this commensurability, we have performed $a b$ initio calculations with VASP [58-62] to check the equilibrium structure of a Tm-ML placed on an 8-ML thick (110) $\mathrm{W}$ slab. The unit cell contains a vacuum thickness twice that of the ML + slab, as customary. Dipolar effects were computationally corrected, too [63-65]. The pseudopotentials include the open-core configurations: [Xe $\left.4 f^{14}\right] 5 p^{6} 5 d^{4} 6 s^{2}$ for $\mathrm{W}$ and $\left[\mathrm{Xe} 4 f^{12}\right] 5 p^{6} 5 d^{1} 6 s^{2}$ for Tm. The cutoff energy was set at an optimal value of $600 \mathrm{eV}$ which warrants a convergence of $<10^{-5} \mathrm{eV}$ for the (110)W slab but just $10^{-2}-10^{-3} \mathrm{eV}$ when the Tm-ML is considered. The Brillouin zone was only sampled at the $\Gamma$ point as a compromise between accuracy and computational efficiency because of the large number of atoms used and the size of the unit cell. After an initial bulk optimization, the cubic W-lattice parameter was set to a relaxed value of $3.180 \AA$, close to the one obtained by other authors [66], while Tm in-plane lattice parameter is fixed by the commensurate structure observed by STM. An optimized structure along the (110) direction of $\mathrm{W}$ was calculated using a quasi-Newton algorithm [67], reducing the HellmannFeynman forces to $\sim 1.0 \mathrm{mRy} / \mathrm{au}$. The average distances between Tm-ML and first $\mathrm{W}$ layer and the $\mathrm{W}$ first and second layers are, respectively, 2.53 and $2.24 \AA$. The latter value is slightly larger than the bulk one, $2.20 \AA$. We obtain a spatial
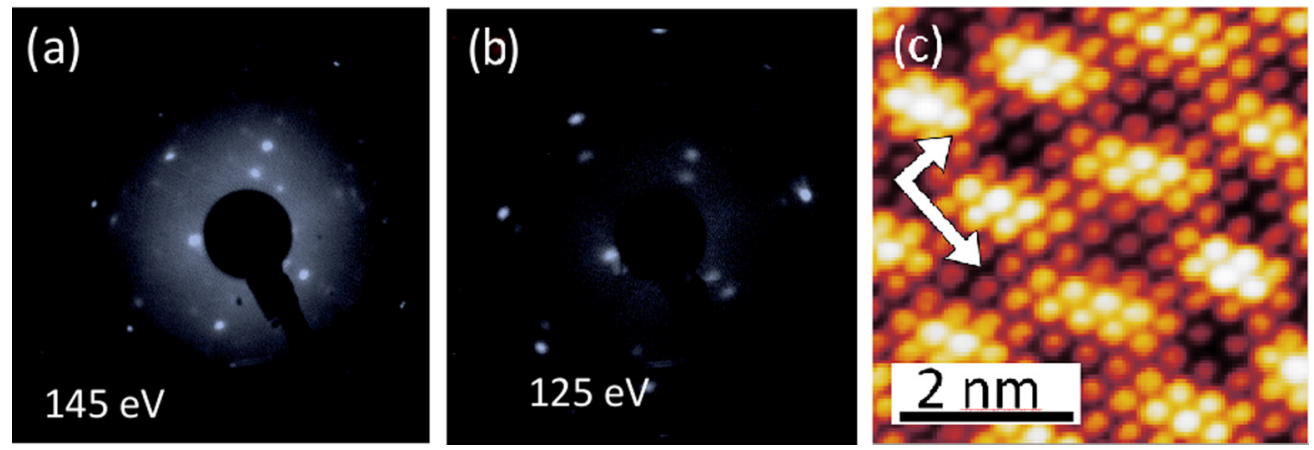

FIG. 2. (Color online) (a) LEED image taken on a Tm deposit showing a characteristic surface reconstruction of rare earth on the (110) W surface, (b) LEED image taken on compact Tm hcp islands coexisting with areas with bare (110) W surface, (c) STM image taken on a terrace covered with Tm; the Moiré pattern can be observed (short and long arrows are the [001] and [110] W crystal directions, respectively). 

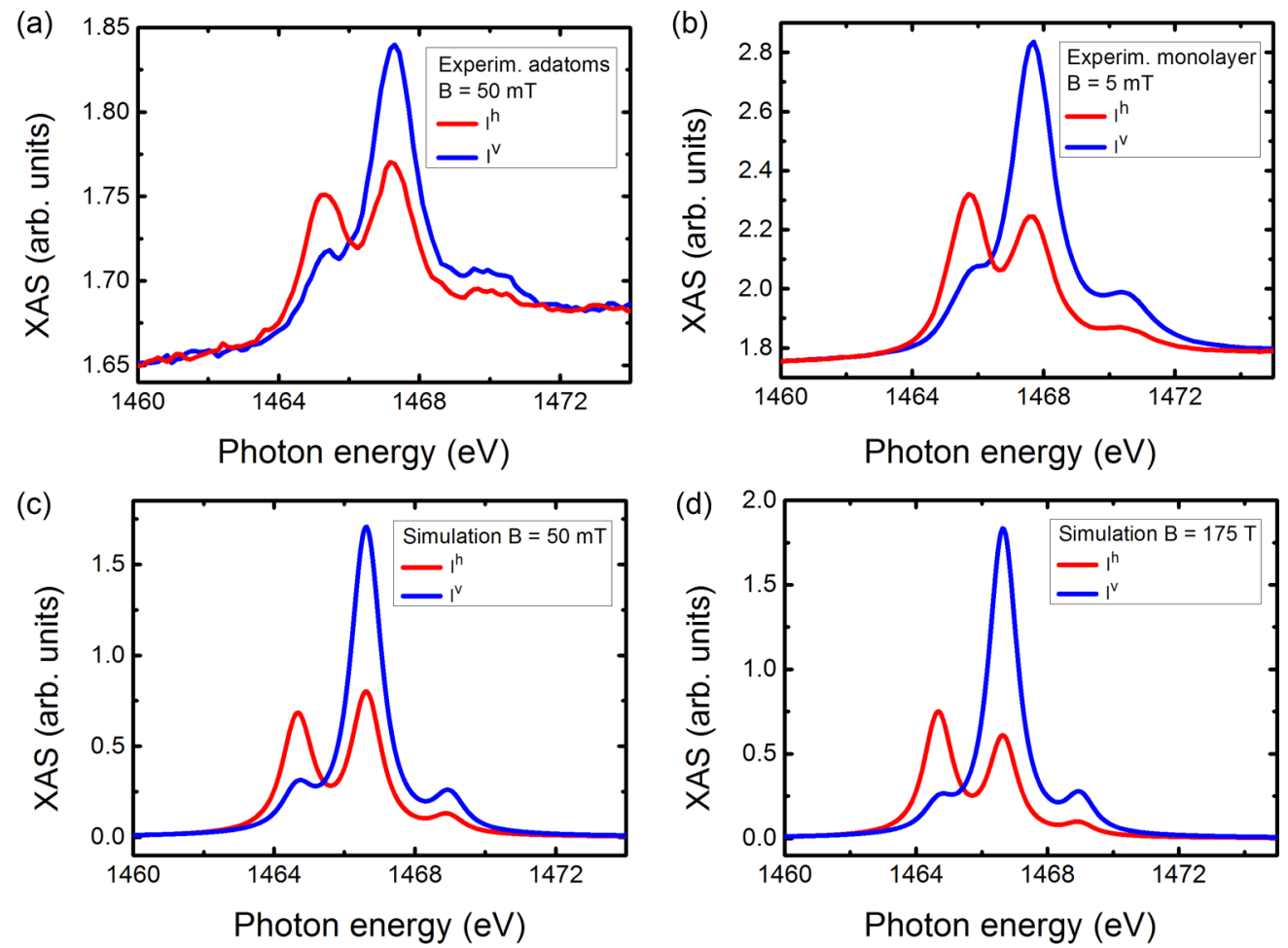

FIG. 3. (Color online) (a) Experimental linearly polarized XAS of Tm adatoms in a 50-mT field; (b) experimental linearly polarized XAS of Tm monolayer in a 5-mT field. (c), (d) Simulated linearly polarized XAS for noninteracting Tm atoms in a 50-mT field applied parallel to the X-ray beam direction and in a 175-T field along the surface normal, respectively. All data are measured/simulated at $T=8 \mathrm{~K}$ with the substrate normal tilted at $70^{\circ}$ with respect to the $\mathrm{x}$-ray beam direction.

peak-to-peak corrugation in the Tm-ML of $0.4 \AA$ with a period of $16 \AA$, both in agreement with the Moiré patterns obtained from STM results. A smaller corrugation also exists in the first-nearest W layer, $\sim 0.05 \AA$, and has about the same spatial periodicity, but it disappears for the next-nearest $\mathrm{W}$ layers.

\section{ELECTRONIC GROUND STATE}

Figure 3 shows the experimental and simulated x-ray absorption spectra for horizontally ( $h$, out-of-plane) and vertically ( $v$, in-plane) polarized light at grazing incidence $\left(\theta=70^{\circ}\right)$. The characteristic multiplet structures of the RE $M_{4,5}$ absorption edges have been widely studied [47,48,68,69] and can be used as a fingerprint for the valence state of the $\mathrm{RE}$ atoms in different compounds. The XAS of the isolated $\mathrm{Tm}$ adatoms as well as the monolayer show typical trivalent absorption features. For the $4 f^{12}$ initial state configuration of $\mathrm{Tm}^{3+}$ the dipole selection rules allow $3 d \rightarrow 4 f$ transitions to $3 d^{9} 4 f^{13}$ final states with ${ }^{3} H_{6},{ }^{3} H_{5}$, and ${ }^{3} G_{5}$ symmetry, giving three absorption lines at the $M_{5}$ edge and a single line at the $M_{4}$ edge, as observed in Fig. 3 (see also Fig. 4). In contrast, a divalent $4 f^{13}$ ground state would give only one absorption line at the $M_{5}$ edge and none at the $M_{4}$ edge $[47,48,68,69]$. This result is somewhat surprising as in the gas phase nearly all RE atoms, with the exception of $\mathrm{La}$ and $\mathrm{Gd}$, are known to exist in a divalent ground state. Moreover, although most of the bulk elemental RE metals are trivalent, Tm forms divalent as well as trivalent and intermediate valence compounds. X-ray absorption and photoemission studies show that divalent Tm atoms are found not only in the gas phase [70], but also in rare gas matrices [71], endohedral fullerenes [72], and at low-coordinated surface sites of Tm films evaporated at low temperature [73]. Here, we find no significant difference in the XAS line shape of isolated adatoms compared to the ML sample, indicating that $\mathrm{Tm}$ atoms on $\mathrm{W}(110)$ have a stable $4 f^{12}$ ground state, which we attribute to the gain in cohesive energy of the $4 f^{12} 5 d^{1} 6 s^{2}$ configuration compared to the $4 f^{13} 5 d^{0} 6 s^{2}$ configuration [74] for Tm atoms adsorbed on a $5 d$ metal [69].

The XAS line-shape analysis indicates a ground-state multiplet with $J=6$. To obtain more detailed information on the ground state of the Tm atoms and take into account the effect of the atomic environment, the XAS spectra in Fig. 3 were simulated in the presence of a crystal-field potential. The substrate was approximated by considering the two lowest-order axial crystal-field components described by the Hamiltonian $H_{\mathrm{CF}}=B_{2}^{0} \hat{O}_{2}^{0}+B_{4}^{0} \hat{O}_{4}^{0}$, where $\hat{O}_{n}^{m}$ are the Stevens operators. The adatom XAS in Fig. 3(a) is well reproduced by using $B_{2}^{0}=-637.2 \mu \mathrm{eV}$ and $B_{4}^{0}=+3.5 \mu \mathrm{eV}$. We find a ground state with $\left|J_{z}\right|=5$; the lowest-excited states are $\left|J_{z}\right|=4$ and 6 , separated by $>9 \mathrm{meV}$ from the ground state. This indicates a predominant uniaxial magnetic anisotropy along the surface normal. The relative intensity of the multiplet features in the linear-polarized XAS of the monolayer differs in comparison with that of the Tm adatoms. In particular, the peak heights of the horizontally polarized XAS are altered. This spectral line shape is neither in accordance with a $\left|J_{z}\right|=6$, nor 4 or any other pure $J_{z}$ ground state, and may indicate a mixed character of the ground state. However, if we assume that 

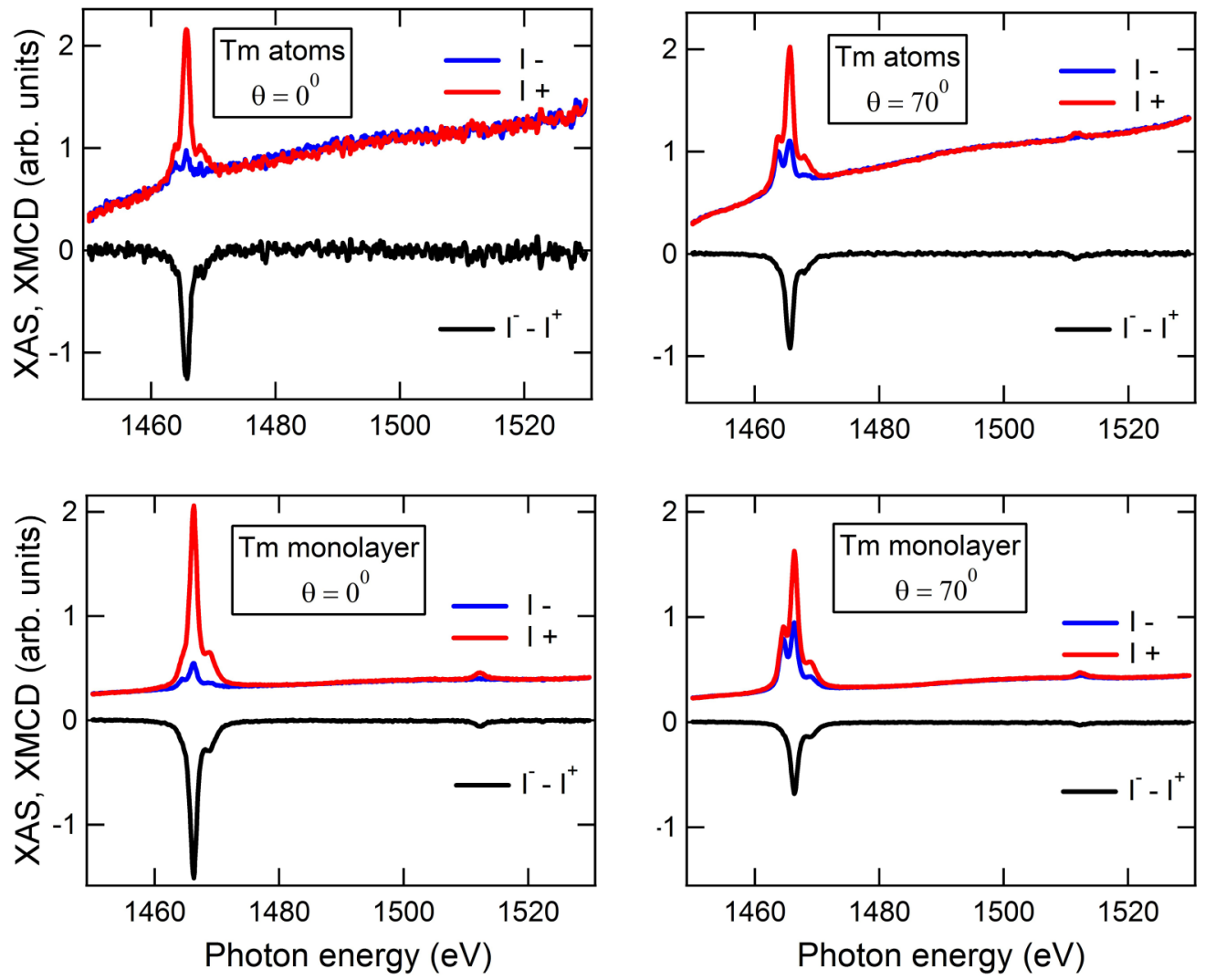

FIG. 4. (Color online) XAS and XMCD spectra of Tm adatoms and monolayers on W(110) measured in a 5-T magnetic field applied parallel to the x-ray direction at $T=8 \mathrm{~K}$. The XMCD spectra are calculated as the difference between $I^{-}$and $I^{+}$.

transversal crystal-field terms that would generate a mixing of different $J_{z}$ states are negligible, the monolayer XAS can be well fitted by applying a strong out-of-plane magnetic field with $B=175 \mathrm{~T}$. Such a strong field may arise from either ferromagnetic or antiferromagnetic exchange coupling within the Tm monolayer, as will be discussed in Sec. VB. The application of a magnetic field leads to the closing of the energy gap between the $J_{z}=-5$ and -6 states with $J_{z}=-5$ still being the lowest state. Thus, the spectral weight of the $J_{z}=-6$ state increases in the absorption and modifies the XAS line shape with respect to the adatom case. This effect is strongest for the horizontal polarization while the vertically polarized XAS remains essentially the same. The temperature dependence of this effect is reported further on.

\section{MAGNETIC CHARACTERIZATION}

\section{A. Magnetic moments and anisotropy}

Figure 4 shows the XAS with circular polarization and corresponding XMCD spectra of the Tm adatom and monolayer samples measured at normal $\left(\theta=0^{\circ}\right)$ and grazing incidence $\left(\theta=70^{\circ}\right)$. The spectra were taken at $8 \mathrm{~K}$ in a 5 - $\mathrm{T}$ magnetic field applied parallel to the $\mathrm{X}$-ray beam direction. The average value of the $M_{5}$ edge jump intensity was normalized to 1 in order to compare the spectra measured on different samples. We note also that the Tm absorption signal is superimposed on the background intensity of the substrate due to the x-ray absorption of $\mathrm{W}$ in this photon energy range.
The sharp multiplet features of the Tm XAS indicate that the spin and orbital magnetic moments must be close to those expected for the $4 f^{12}$ ground state, namely, 2 and $5 \mu_{B}$ /atom, respectively [75]. This is confirmed by the very large XMCD asymmetry measured at $8 \mathrm{~K}$ and by the orbital-to-spin moment ratio estimated from the XMCD sum rules. For the RE, this ratio is given by $[76,77]$

$$
\frac{\left\langle L_{z}\right\rangle}{2\left\langle S_{z}\right\rangle}=\frac{X_{M_{5}}+X_{M_{4}}}{X_{M_{5}}-\frac{3}{2} X_{M_{4}}}\left(1+3 \frac{\left\langle T_{z}\right\rangle}{\left\langle S_{z}\right\rangle}\right),
$$

where $\left\langle L_{z}\right\rangle,\left\langle S_{z}\right\rangle$, and $\left\langle T_{z}\right\rangle$ are the expectation values of the orbital, spin, and magnetic dipole moments parallel to the X-ray direction, and $X_{M_{4}}\left(X_{M_{5}}\right)$ represent the XMCD intensity integrated over the $M_{4}\left(M_{5}\right)$ energy edge, respectively. For both the adatom and the monolayer samples, taking the ratio $\left\langle T_{z}\right\rangle /\left\langle S_{z}\right\rangle=0.41$ calculated for $\mathrm{Tm}^{3+}$ (Ref. [75]), we obtain $\left\langle L_{z}\right\rangle / 2\left\langle S_{z}\right\rangle=2.5 \pm 0.1$.

Figure 4 also shows that the XMCD amplitude is larger at normal incidence compared to grazing incidence. This implies that, for equal applied magnetic fields, the Tm magnetization is larger at normal incidence, i.e., that both the Tm adatoms and the monolayers have an out-of-plane easy axis, as expected from the results of the ligand field multiplet calculations presented in Sec. IV. Magnetization loops were measured by recording the peak XMCD intensity at the $M_{5}$ edge at different magnetic fields. Figure 5 shows the field dependence of the XMCD of the Tm adatom and monolayer samples at normal and grazing incidence. The larger amplitude of the XMCD loops at normal incidence agrees with the easy magnetization 

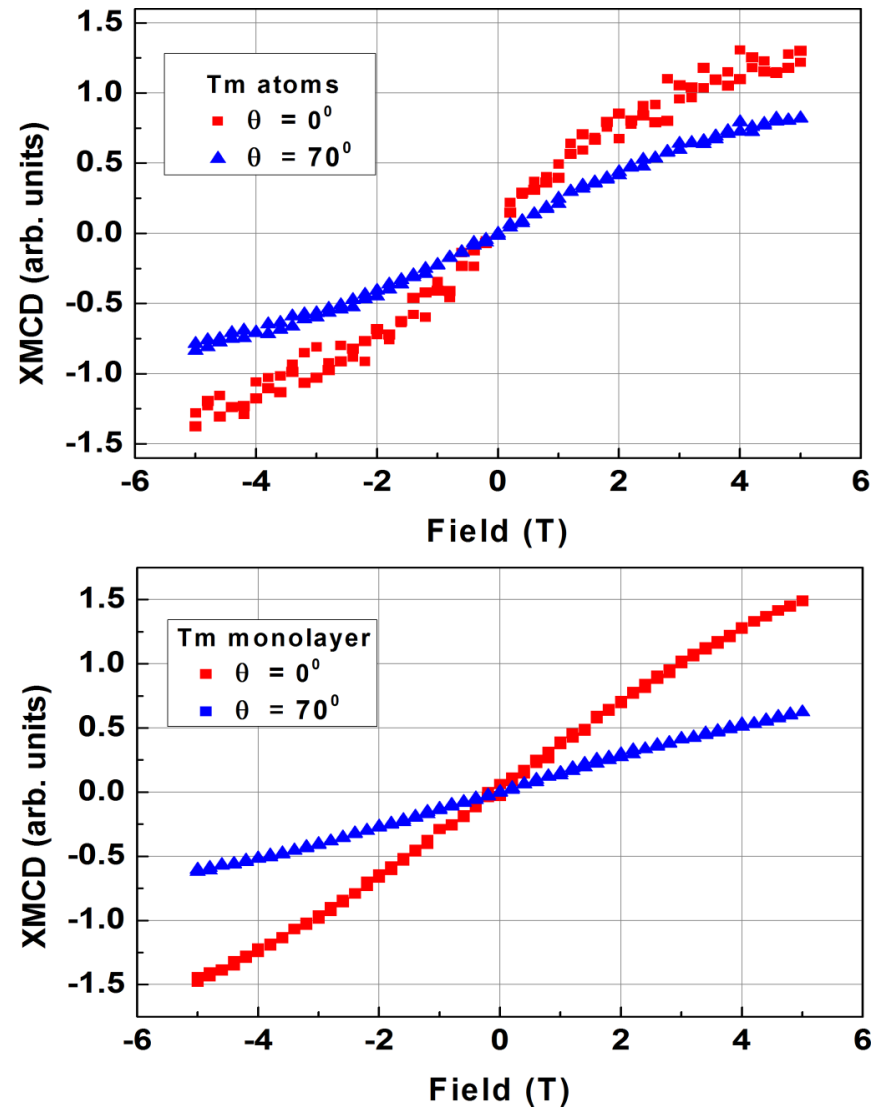

FIG. 5. (Color online) Field dependence of the Tm XMCD intensity measured at the $M_{5}$ edge for the adatom and the monolayer samples at $8 \mathrm{~K}$.

axis being perpendicular to the substrate for both the $\mathrm{Tm}$ adatoms and the Tm monolayers. The comparison between the two curves, however, is not straightforward. According to the XMCD sum rules, the $M_{5} \mathrm{XMCD}$ intensity is proportional to $4\left\langle S_{\theta}\right\rangle+3\left\langle L_{\theta}\right\rangle+12\left\langle T_{\theta}\right\rangle$, where we recall that $\theta$ is the direction of the x-ray beam. For a given $\theta$, since $S, L$, and $T$ are strongly coupled together, this quantity is proportional to the Tm magnetization. Due to the strong anisotropy of $L$ and $T$ in the RE, the proportionality factors between the magnetization $\left(2\left\langle S_{\theta}\right\rangle+\left\langle L_{\theta}\right\rangle\right)$ and the $M_{5}$ XMCD intensity measured at $\theta=0^{\circ}$ and $70^{\circ}$ can differ significantly, which makes it difficult, e.g., to estimate the magnetic anisotropy energy from such curves. Moreover, the RKKY interaction among adatoms, which we cannot quantify here, may further affect the magnetization behavior $[4,78]$.

\section{B. Magnetism of monolayer films}

The nonhysteretic shape of the magnetization curves indicates the absence of either ferromagnetic or ferrimagnetic ordering in both samples over this range of experimental conditions. Figure 5, moreover, shows that the normal incidence and grazing incidence XMCD curves of the Tm monolayer have a less pronounced $\mathrm{S}$ shape compared to the adatoms, contrary to what would be expected for a superparamagnetic system. This suggests the presence of an additional antiferromagnetic coupling in the Tm monolayer. To further investigate the

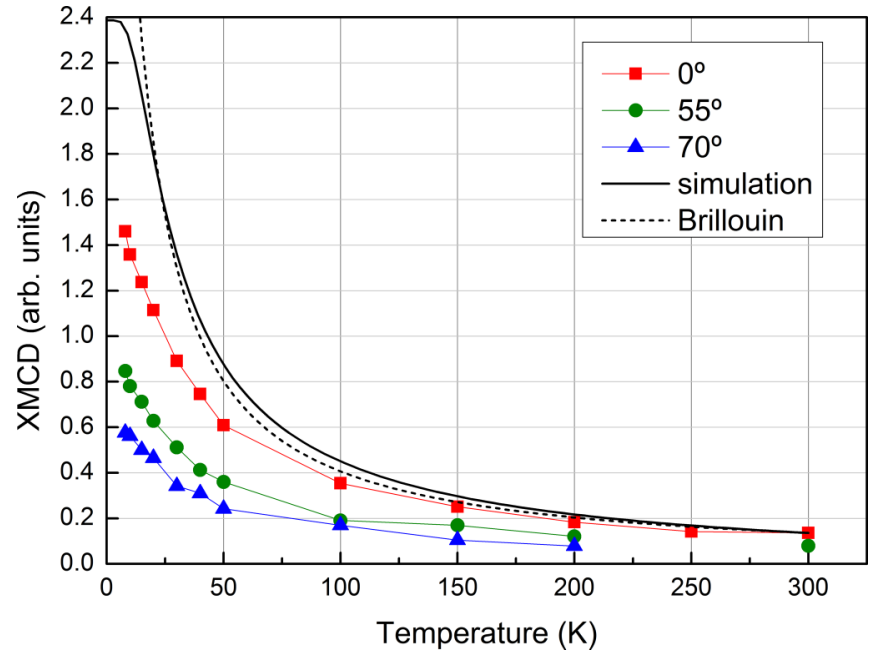

FIG. 6. (Color online) Tm monolayer XMCD as a function of temperature (dots) compared to the paramagnetic behavior expected for noninteracting $\mathrm{Tm}$ atoms (lines). The experimental data were taken in a 5-T magnetic applied parallel to the x-ray beam, at $\theta=$ $0^{\circ}, 55^{\circ}$, and $70^{\circ}$. The paramagnetic curves were calculated using a spin Hamiltonian model and crystal-field parameters obtained from the multiplet simulations (solid line) and by assuming a Brillouin function with the $\mathrm{Tm}^{3+}$ parameters $J=6$ and $g=\frac{7}{6}$ (dashed line). The calculated curves are normalized to the experimental data at $300 \mathrm{~K}$.

possibility of magnetic interactions in the Tm monolayer, we acquired Tm XMCD spectra while cooling down the sample in a magnetic field of $5 \mathrm{~T}$ and plotted the XMCD intensity at the $M_{5}$ edge as a function of temperature in Fig. 6 . The experimental data are compared with simulations of the $\mathrm{Tm}$ magnetization according to a spin Hamiltonian model taking into account the crystal-field parameters $B_{2}^{0}$ and $B_{4}^{0}$ (Sec. IV) and a Brillouin function calculated for $J=6$ and a magnetic field of $5 \mathrm{~T}$. In either case, we notice a departure of the experimental magnetization from the predicted paramagnetic dependence that becomes prominent at temperatures below $100 \mathrm{~K}$, which suggests the setting in of magnetic correlations around this temperature. Since the experimental magnetization in this low-temperature range is lower than predicted by the paramagnetic simulations, we can infer that the Tm-Tm interactions are antiferromagnetic.

To confirm the tendency to antiferromagnetic correlations of the Tm monolayer, we also measured the temperature dependence of X-ray magnetic linear dichroism (XMLD) spectra. As opposed to XMCD, which probes unidirectional magnetic ordering, XMLD is sensitive to uniaxial magnetic ordering, and therefore well suited to detect antiferromagnetic coupling [79]. Figures 7(a)-7(c) show the $M_{5}$ edge linearly polarized XAS measured at three different temperatures. For the spectra labeled $I^{v}$ (vertical polarization), the photons are polarized parallel to the sample plane and therefore these spectra are sensitive to changes in the in-plane magnetic ordering of the Tm monolayer. For the spectra labeled $I^{h}$ (horizontal polarization), the photons have a finite linear polarization component along the sample normal, and therefore are sensitive to changes in the out-of-plane magnetic ordering of the $\mathrm{Tm}$ monolayer. 

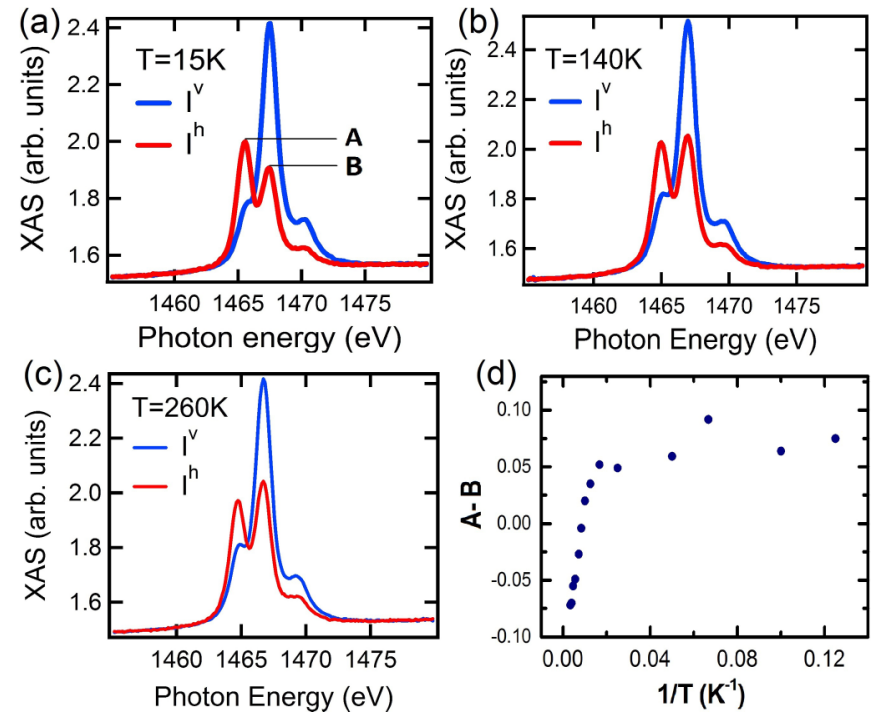

FIG. 7. (Color online) (a)-(c) Linearly polarized XAS spectra measured on the Tm monolayer at three different temperatures. All spectra were taken with the substrate normal tilted at $\theta=70^{\circ}$ with respect to the x-ray beam direction. A small (5-mT) magnetic field was applied to enhance the electron yield of the sample without significantly affecting its magnetic configuration. The first and second peaks in the $h$-polarized spectra are labeled A and B, respectively. (d) Temperature dependence of the difference between $\mathrm{A}$ and $\mathrm{B}$ peaks.

Whereas the $v$ spectra change little with temperature, there is a notable temperature dependence of the $h$ spectra. To quantify this temperature dependence, we plot in Fig. 7(d) the difference between the first and the second peaks in the $h$ spectra [A and B in Fig. 7(a)] as a function of temperature. The difference between the two peak values increases with $1 / T$ at high temperatures and reaches a saturation value at about $50 \mathrm{~K}$, suggesting that antiferromagnetic correlations are well established at this temperature. The fact that these changes occur in the $h$ spectra suggests that the axis of antiferromagnetic ordering is parallel to the sample normal, in agreement with the easy-axis direction determined from the magnetization curves in Fig. 5. This conclusion is further supported by the $I^{h}$ and $I^{v}$ spectra simulated by considering noninteracting adatoms and an out-of-plane magnetic field of $B=175 \mathrm{~T}$, which we attribute to antiferromagnetic exchange. As shown in Figs. 3(c) and 3(d), the application of such a strong field reproduces the change of intensity of the A and B peaks observed upon cooling.

It is instructive to compare the magnetic behavior of Tm monolayers on W(110) with that of bulk Tm. Neutron diffraction and ac susceptibility studies [80] have shown that bulk Tm undergoes an antiferromagnetic transition at $T=58 \mathrm{~K}$, followed by a transition to ferrimagnetic ordering at $T=33 \mathrm{~K}$. These low magnetic ordering temperatures are attributed to the strong localization of the $4 f$ electrons, which makes the exchange interaction with neighboring atoms small. Interactions with the neighbors still occur, but indirectly via $s$ and $p$ electrons, which favor antiferromagnetic ordering [43]. Between 58 and $33 \mathrm{~K}$, the bulk Tm moments are aligned ferromagnetically within the hcp basal planes and have a sinusoidal modulation along the $c$ axis, such that the ordering is antifer- romagnetic along the $c$ axis. As opposed to bulk Tm crystals, the Tm monolayers studied here display antiferromagnetic coupling within the basal plane. Antiferromagnetic coupling is favored parallel to the substrate normal by the perpendicular magnetic anisotropy generated through hybridization with the electronic states of the $\mathrm{W}(110)$ substrate.

The presence of antiferromagnetic coupling within the plane of the $\mathrm{Tm}$ monolayer is therefore intriguing. It also raises a number of questions regarding the magnetic ground state of this system. Because the Tm lattice is (nearly) hexagonal, as shown in Sec. III, geometric frustration is expected to play an important role. In fact, there is no unique ground state for a triangular arrangement of magnetic moments coupled antiferromagnetically with each other $[81,82]$. Depending on the relative value of the exchange coupling, magnetic anisotropy, and external field, several configurations are possible such as, e.g., one spin pointing up and two pointing down at an angle or collinear with each other or a canted spin structure with a net perpendicular component aligned along the field direction [83]. This may explain why the magnetic response of the Tm monolayer is larger when the magnetic field is applied perpendicular to the substrate compared to grazing incidence [Figs. 5(b) and 6], opposite to that of an isotropic antiferromagnet for which the field-induced magnetization is larger in the direction perpendicular to the antiferromagnetic axis. Moreover, owing to the large degeneracy of the magnetic ground state, frustration in two-dimensional systems may also lead to an intrinsically disordered ground state in the absence of an external field. This is the case for a two-dimensional triangular antiferromagnet of the Ising type [81]. Lattice distortions and defects that affect the exchange interaction between neighboring magnetic ions lead to a partial lifting of frustration. However, even in such a case, frustration will induce a suppression of the ordering temperature below that corresponding to the energy scale of the exchange interaction, so that a correlated antiferromagnetic state persists in the absence of magnetic order. Our observations of triangular lattice structure, antiferromagnetic spin alignment at low temperature, uniaxial anisotropy, and lack of magnetic remanence for Tm monolayer films are consistent with this picture, although we cannot exclude alternative explanations based on Tm domains with fully compensated staggered magnetization or noncollinear structures due to the influence of the Dzyaloshinskii-Moriya interaction at the $\mathrm{Tm} / \mathrm{W}(110)$ interface $[84,85]$. Distinguishing between these possibilities requires the use of spatially resolved or magnetic scattering techniques, which go beyond the scope of this work.

\section{CONCLUSIONS}

We have characterized the growth of Tm adatoms and monolayers on $\mathrm{W}(110)$, showing that isolated atoms are obtained at low coverage $(\sim 2 \%)$ and annealing below $1000 \mathrm{~K}$, whereas heteroepitaxial $\mathrm{Tm} / \mathrm{W}(110)$ monolayers are obtained at high coverage upon annealing to $1100 \mathrm{~K}$. The Tm monolayer exhibits a distorted hexagonal structure, which is compressed along the [110] W direction by $1 \%$ and expanded along the [100] W direction by $15 \%$ with respect to the bulk hop structure of Tm. 
We measured linear and circular x-ray magnetic dichroism to investigate the electronic structure and magnetic behavior of $\mathrm{Tm}$ atoms and monolayers. We show that Tm atoms present a $4 f^{12}$ ground state as isolated impurities as well as in monolayer films with correspondingly large spin and orbital magnetic moments. The orbital-to-spin magnetic moment ratio is 2.5 , as expected for a $J=6$ ground multiplet with $L=5$ and $S=1$. By comparing the experimental XAS with ligand field multiplet calculations, we find that the Tm ground state has $\left|J_{z}\right|=5$; the lowest-excited states correspond to $\left|J_{z}\right|=4$ and 6, separated by more than $9 \mathrm{meV}$ from the ground state. The Tm atoms and monolayers exhibit out-of-plane magnetic anisotropy and nonhysteretic magnetization curves down to $8 \mathrm{~K}$.

Temperature-dependent measurements of magnetic linear and circular dichroism further show that the Tm monolayers develop strong antiferromagnetic correlations at a temperature of about $50 \mathrm{~K}$. The temperature-induced changes of the linearly polarized spectra as well as multiplet simulations including an effective exchange field show that the antiferromagnetic axis is perpendicular to the substrate, in agreement with the sign of the uniaxial anisotropy. This behavior differs from that of classical antiferromagnetic systems, which show a maximum of the magnetization at $T_{N}$ and a larger susceptibility perpendicular to the easy axis below $T_{N}$. Our measurements do not allow us to conclude whether the system exhibits longrange staggered antiferromagnetic order or a disordered state with antiferromagnetic correlations. However, the triangular structure of the Tm lattice suggests that there may be a significant degree of magnetic frustration in this system.

\section{ACKNOWLEDGMENTS}

This work was supported by the European Research Council (StG 203239 NOMAD), Agència de Gestió d'Ajuts Universitaris i de Recerca (2009 SGR 695), Spanish MINECO (Grants No. MAT2009-10040, No. MAT2010-15659, and No. MAT2012-31309), Gobierno de Aragón (Grant No. E81), Fondo Social Europeo, and the Swiss Competence Centre for Materials Science and Technology (CCMX). The computer resources used at the cluster "Memento" within the National Center of Supercomputation, Project No. QCM2013-2-0031, and assistance provided by A. Giner and G. Losilla of the BIFI at the University of Zaragoza are thankfully acknowledged.
[1] J. Li, W.-D. Schneider, R. Berndt, and B. Delley, Phys. Rev. Lett. 80, 2893 (1998).

[2] S. Gardonio, T. O. Wehling, L. Petaccia, S. Lizzit, P. Vilmercati, A. Goldoni, M. Karolak, A. I. Lichtenstein, and C. Carbone, Phys. Rev. Lett. 107, 026801 (2011).

[3] T. Schuh, T. Miyamachi, S. Gerstl, M. Geilhufe, M. Hoffmann, S. Ostanin, W. Hergert, A. Ernst, and W. Wulfhekel, Nano Lett. 12, 4805 (2012).

[4] C.-Y. Lin, J.-L. Li, Y.-H. Hsieh, K.-L. Ou, and B. A. Jones, Phys. Rev. X 2, 021012 (2012).

[5] T. Miyamachi, T. Schuh, T. Märkl, C. Bresch, T. Balashov, A. Stöhr, C. Karlewski, S. André, M. Marthaler, M. Hoffmann et al., Nature (London) 503, 242 (2013).

[6] C. Rau, C. Jin, and M. Robert, Phys. Lett. A 138 (1989).

[7] M. Farle, K. Baberschke, U. Stetter, A. Aspelmeier, and F. Gerhardter, Phys. Rev. B 47, 11571 (1993).

[8] L. Berbil-Bautista, S. Krause, M. Bode, and R. Wiesendanger, Phys. Rev. B 76, 064411 (2007).

[9] M. Getzlaff, Surface Magnetism, Vol. 240 of Springer Tracts in Modern Physics (Springer, Berlin, 2010), pp. 21-52.

[10] E. Weschke, H. Ott, E. Schierle, C. Schüßler Langeheine, D. Vyalikh, G. Kaindl, V. Leiner, M. Ay, T. Schmitte, H. Zabel et al., Phys. Rev. Lett. 93, 157204 (2004).

[11] N. Sato, J. Appl. Phys. 59, 2514 (1986).

[12] S. Maat, N. Smith, M. J. Carey, and J. R. Childress, Appl. Phys. Lett. 93, 103506 (2008).

[13] M. K. Marcham, W. Yu, P. S. Keatley, L. R. Shelford, P. Shafer, S. A. Cavill, H. Qing, A. Neudert, J. R. Childress, J. A. Katine et al., Appl. Phys. Lett. 102, 062418 (2013).

[14] B. Sanyal, C. Antoniak, T. Burkert, B. Krumme, A. Warland, F. Stromberg, C. Praetorius, K. Fauth, H. Wende, and O. Eriksson, Phys. Rev. Lett. 104, 156402 (2010).

[15] C. Ward, G. Scheunert, W. R. Hendren, R. Hardeman, M. A. Gubbins, and R. M. Bowman, Appl. Phys. Lett. 102, 092403 (2013).
[16] J. J. Rhyne, R. W. Erwin, J. Borchers, S. Sinha, M. B. Salamon, R. Du, and C. P. Flynn, J. Appl. Phys. 61, 4043 (1987).

[17] J. Borchers, S. Sinha, M. B. Salamon, R. Du, C. P. Flynn, J. J. Rhyne, and R. W. Erwin, J. Appl. Phys. 61, 4049 (1987).

[18] P. Dowben, D. McIlroy, and D. Li, Handbook Phys. Chem. Rare Earths 24, 1 (1997).

[19] L. Benito, J. I. Arnaudas, M. Ciria, C. de la Fuente, A. del Moral, R. C. C. Ward, and M. R. Wells, Phys. Rev. B 70, 052403 (2004).

[20] G. Bergmann, Phys. Rev. B 23, 3805 (1981).

[21] P. Gambardella, S. S. Dhesi, S. Gardonio, C. Grazioli, P. Ohresser, and C. Carbone, Phys. Rev. Lett. 88, 047202 (2002).

[22] P. Gambardella, S. Rusponi, M. Veronese, S. S. Dhesi, C. Grazioli, A. Dallmeyer, I. Cabria, R. Zeller, P. H. Dederichs, K. Kern et al., Science 300, 1130 (2003).

[23] P. Gambardella, H. Brune, S. S. Dhesi, P. Bencok, S. R. Krishnakumar, S. Gardonio, M. Veronese, C. Grazioli, and C. Carbone, Phys. Rev. B 72, 045337 (2005).

[24] J. Honolka, K. Kuhnke, L. Vitali, A. Enders, K. Kern, S. Gardonio, C. Carbone, S. R. Krishnakumar, P. Bencok, S. Stepanow et al., Phys. Rev. B 76, 144412 (2007).

[25] H. Brune and P. Gambardella, Surf. Sci. 603, 1812 (2009).

[26] C. Carbone, M. Veronese, P. Moras, S. Gardonio, C. Grazioli, P. H. Zhou, O. Rader, A. Varykhalov, C. Krull, T. Balashov et al., Phys. Rev. Lett. 104, 117601 (2010).

[27] A. J. Heinrich, J. A. Gupta, C. P. Lutz, and D. M. Eigler, Science 306, 466 (2004).

[28] C. F. Hirjibehedin, C.-Y. Lin, A. F. Otte, M. Ternes, C. P. Lutz, B. A. Jones, and A. J. Heinrich, Science 317, 1199 (2007).

[29] A. A. Khajetoorians, S. Lounis, B. Chilian, A. T. Costa, L. Zhou, D. L. Mills, J. Wiebe, and R. Wiesendanger, Phys. Rev. Lett. 106, 037205 (2011).

[30] C. Carbone, R. Rochow, L. Braicovich, R. Jungblut, T. Kachel, D. Tillmann, and E. Kisker, Phys. Rev. B 41, 3866 (1990). 
[31] F. Hübinger, C. Schüßler Langeheine, A. Fedorov, K. Starke, E. Weschke, A. Höhr, S. Vandré, and G. Kaindl, J. Electron Spectrosc. Relat. Phenom. 76, 535 (1995).

[32] O. Paul, S. Toscano, W. Hürsch, and M. Landolt, J. Magn. Magn. Mater. 84, L7 (1990).

[33] R. Kappert, M. Sacchi, J. Goedkoop, M. Grioni, and J. Fuggle, Surf. Sci. 248, L245 (1991).

[34] R. J. H. Kappert, J. Vogel, M. Sacchi, and J. C. Fuggle, Phys. Rev. B 48, 2711 (1993).

[35] J. Vogel and M. Sacchi, Surf. Sci. 365, 831 (1996).

[36] M. Getzlaff, M. Bode, S. Heinze, R. Pascal, and R. Wiesendanger, J. Magn. Magn. Mater. 184, 155 (1998).

[37] M. Getzlaff, M. Bode, S. Heinze, and R. Wiesendanger, Appl. Surf. Sci. 142, 558 (1999).

[38] M. Bode, M. Getzlaff, A. Kubetzka, R. Pascal, O. Pietzsch, and R. Wiesendanger, Phys. Rev. Lett. 83, 3017 (1999).

[39] D. Weller, S. F. Alvarado, W. Gudat, K. Schröder, and M. Campagna, Phys. Rev. Lett. 54, 1555 (1985).

[40] M. Taborelli, R. Allenspach, G. Boffa, and M. Landolt, Phys. Rev. Lett. 56, 2869 (1986).

[41] C. A. F. Vaz, J. A. C. Bland, and G. Lauhoff, Rep. Prog. Phys. 71, 056501 (2008).

[42] U. Gradmann, in Handbook of Magnetic Materials, Vol. 7, edited by K. H. J. Buschow (Elsevier, Amsterdam, 1993), Chap. 1, pp. 1-96.

[43] J. Jensen and A. Mackintosh, Rare Earth Magnetism: Structures and Excitations (Clarendon Press, Oxford, 1991).

[44] M. Bode, S. Krause, L. Berbil-Bautista, S. Heinze, and R. Wiesendanger, Surf. Sci. 601, 3308 (2007).

[45] M. Ternes, M. Pivetta, F. Patthey, and W.-D. Schneider, Prog. Surf. Sci. 85, 1 (2010).

[46] J. Diez-Ferrer, D. Coffey, M. Ciria, and J. I. Arnaudas (unpublished).

[47] B. T. Thole, G. van der Laan, J. C. Fuggle, G. A. Sawatzky, R. C. Karnatak, and J.-M. Esteva, Phys. Rev. B 32, 5107 (1985).

[48] J. B. Goedkoop, B. T. Thole, G. van der Laan, G. A. Sawatzky, F. M. F. de Groot, and J. C. Fuggle, Phys. Rev. B 37, 2086 (1988).

[49] S. Stepanow, A. Mugarza, G. Ceballos, P. Moras, J. C. Cezar, C. Carbone, and P. Gambardella, Phys. Rev. B 82, 014405 (2010).

[50] I. G. Rau, S. Baumann, S. Rusponi, F. Donati, S. Stepanow, L. Gragnaniello, J. Dreiser, C. Piamonteze, F. Nolting, S. Gangopadhyay, O. R. Albertini, R. M. Macfarlane, C. P. Lutz, B. A. Jones, P. Gambardella, A. J. Heinirich, and H. Brune, Science 344, 988 (2014).

[51] J. Kolaczkiewicz and E. Bauer, Surf. Sci. 175, 487 (1986).

[52] C. Nicklin, C. Binns, C. Norris, P. McCluskey, and M.-G. Barthés-Labrousse, Surf. Sci. 269-270, 700 (1992).

[53] M. Bodenbach, A. Hohr, C. Laubschat, G. Kaindl, and M. Methfessel, Phys. Rev. B 50, 14446 (1994).

[54] A. Stenborg and E. Bauer, Phys. Rev. B 36, 5840 (1987).

[55] J. Koaczkiewicz, Vacuum 56, 191 (2000).
[56] H. Li, D. Tian, J. Quinn, Y. S. Li, S. C. Wu, and F. Jona, Phys. Rev. B 45, 3853 (1992).

[57] E. D. Tober, R. X. Ynzunza, C. Westphal, and C. S. Fadley, Phys. Rev. B 53, 5444 (1996).

[58] G. Kresse and J. Furthmüller, Phys. Rev. B 54, 11169 (1996).

[59] G. Kresse and J. Furthmuller, Comput. Mater. Sci. 6, 15 (1996).

[60] P. E. Blöchl, Phys. Rev. B 50, 17953 (1994).

[61] G. Kresse and D. Joubert, Phys. Rev. B 59, 1758 (1999).

[62] J. P. Perdew, J. A. Chevary, S. H. Vosko, K. A. Jackson, M. R. Pederson, D. J. Singh, and C. Fiolhais, Phys. Rev. B 46, 6671 (1992).

[63] G. Makov and M. C. Payne, Phys. Rev. B 51, 4014 (1995).

[64] J. Neugebauer and M. Scheffler, Phys. Rev. B 46, 16067 (1992).

[65] X. Qian and W. Hübner, Phys. Rev. B 60, 16192 (1999).

[66] D. Spisak and J. Hafner, Phys. Rev. B 70, 195426 (2004).

[67] P. Pulay, Chem. Phys. Lett. 73, 393 (1980).

[68] J. Sugar, Phys. Rev. A 6, 1764 (1972).

[69] G. Kaindl, G. Kalkowski, W. D. Brewer, B. Perscheid, and F. Holtzberg, J. Appl. Phys. 55, 1910 (1984).

[70] B. K. Sarpal, C. Blancard, J. P. Connerade, J. M. Esteva, J. Hormes, R. C. Karnatak, and U. Kuetgens, J. Phys. B: At., Mol. Opt. Phys. 24, 1593 (1991).

[71] C. Blancard, J. M. Esteva, J. P. Connerade, U. Kuetgens, and J. Hormes, J. Phys. B: At., Mol. Opt. Phys. 22, L575 (1989).

[72] T. Pichler, M. S. Golden, M. Knupfer, J. Fink, U. Kirbach, P. Kuran, and L. Dunsch, Phys. Rev. Lett. 79, 3026 (1997).

[73] M. Domke, C. Laubschat, M. Prietsch, T. Mandel, G. Kaindl, and W. D. Schneider, Phys. Rev. Lett. 56, 1287 (1986).

[74] B. Johansson, Phys. Rev. B 19, 6615 (1979).

[75] Y. Teramura, A. Tanaka, B. T. Thole, and T. Jo, J. Phys. Soc. Jpn. 65, 3056 (1996).

[76] B. T. Thole, P. Carra, F. Sette, and G. van der Laan, Phys. Rev. Lett. 68, 1943 (1992).

[77] P. Carra, B. T. Thole, M. Altarelli, and X. Wang, Phys. Rev. Lett. 70, 694 (1993).

[78] L. Zhou, J. Wiebe, S. Lounis, E. Vedmedenko, F. Meier, S. Blügel, P. H. Dederichs, and R. Wiesendanger, Nat. Phys. 6, 187 (2010).

[79] B. T. Thole, G. van der Laan, and G. A. Sawatzky, Phys. Rev. Lett. 55, 2086 (1985).

[80] H. Astrom, J. Nogues, G. Nicolaides, K. Rao, and G. Benediktsson, J. Phys.: Condens. Matter 3, 7395 (1991).

[81] G. H. Wannier, Phys. Rev. 79, 357 (1950).

[82] P. Fazekas and P. Anderson, Philos. Mag. 30, 423 (1974).

[83] M. Collins and O. Petrenko, Can. J. Phys. 75, 605 (1997).

[84] M. Bode, M. Heide, K. Von Bergmann, P. Ferriani, S. Heinze, G. Bihlmayer, A. Kubetzka, O. Pietzsch, S. Blügel, and R. Wiesendanger, Nature (London) 447, 190 (2007).

[85] S. V. Grigoriev, Y. O. Chetverikov, D. Lott, and A. Schreyer, Phys. Rev. Lett. 100, 197203 (2008). 